\title{
Roudneff's Conjecture for Lawrence Oriented Matroids
}

\author{
Luis Pedro Montejano \\ Institut de Mathématiques et de Modélisation de Montpellier \\ Université de Montpellier \\ Montpellier, France \\ lpmontejano@gmail.com \\ Jorge Luis Ramírez Alfonsín * \\ Institut de Mathématiques et de Modélisation de Montpellier \\ Université de Montpellier \\ Montpellier, France \\ jramirez@um2.fr
}

Submitted: Nov 4, 2014; Accepted: Mar 19, 2015; Published: Apr 14, 2015

Mathematics Subject Classifications: 52C40, 05C35

\begin{abstract}
J.-P. Roudneff has conjectured that every arrangement of $n \geqslant 2 d+1 \geqslant 5$ (pseudo) hyperplanes in the real projective space $\mathbb{P}^{d}$ has at most $\sum_{i=0}^{d-2}\left(\begin{array}{c}n-1 \\ i\end{array}\right)$ cells bounded by each hyperplane. In this note, we show the validity of this conjecture for arrangements arising from Lawrence oriented matroids.
\end{abstract}

Keywords: Lawrence Oriented Matroids, Arrangements of Hyperplanes.

\section{Introduction}

An Euclidean (resp. projective) $d$-arrangement of $n$ hyperplanes $H(d, n)$ is a finite collection of hyperplanes in the Euclidean space $\mathbb{R}^{d}$ (resp. the real projective space $\mathbb{P}^{d}$ ) such that no point belongs to every hyperplane of $H(d, n)$. Any arrangement $H$ decomposes $\mathbb{R}^{d}$ (resp. $\mathbb{P}^{d}$ ) into a $d$-dimensional cell complex. It is clear that any $d$-cell $c$ of $H(d, n)$ has at most $n$ facets (that is, $(d-1)$-cells). We say that $c$ is a complete cell of $H$ if $c$ has exactly $n$ facets, i.e., $c$ is bounded by each hyperplane of $H(d, n)$.

*This work was partially supported by the ANR TEOMATRO grant ANR-10-BLAN 0207 and by the PICS's project PICS06316. 
The cyclic polytope of dimension $d$ with $n$ vertices $C_{d}\left(t_{1}, \ldots, t_{n}\right)$, which was discovered by Carathéodory [2], is the convex hull in $\mathbb{R}^{d}, d \geqslant 2$ of $n \geqslant d+1$ different points $x\left(t_{1}\right), \ldots, x\left(t_{n}\right)$ of the moment curve $x: \mathbb{R} \longrightarrow \mathbb{R}^{d}, t \mapsto\left(t, t^{2}, \ldots, t^{d}\right)$. Cyclic polytopes play an important role in the combinatorial convex geometry due to their connection with certain extremal problems, for example, the upper bound theorem due to McMullen [7]. Cyclic arrangements are defined as the dual of the cyclic polytopes. As for cyclic polytopes, cyclic arrangements also have extremal properties. For instance, Shannon [12] has introduced cyclic arrangements on dimension $d$ as examples of projective arrangements with a minimum number of cells with $(d+1)$-facets.

In [10] Roudneff proved that the number of complete cells of the cyclic arrangements on dimension $d$ with $n$ hyperplanes, denoted as $f(d, n)$, is at least $\sum_{i=0}^{d-2}\left(\begin{array}{c}n-1 \\ i\end{array}\right)$. Roudneff showed that the latter is tight for all $n \geqslant 2 d+1$ and conjectured that, in this case, cyclic arrangements have the maximum number of complete cells.

Conjecture 1. [10, Conjecture 2.2] Every arrangement of $n \geqslant 2 d+1 \geqslant 5$ (pseudo) hyperplanes in $\mathbb{P}^{d}$ has at most $\sum_{i=0}^{d-2}\left(\begin{array}{c}n-1 \\ i\end{array}\right)$ complete cells.

It is known that Conjecture 1 is true for $d=2$ (that is, any arrangement of $n$ pseudolines in $\mathbb{P}^{2}$ contains at most one complete cell). Ramírez Alfonsín [8] proved the conjecture for the case $d=3$.

Forge and Ramírez Alfonsín [4] calculated the exact number of complete cells of cyclic arrangements for any positive integers $d$ and $n$ such that $n \geqslant d+1$, namely,

$$
f(d, n)=\sum_{i=0}^{d-2}\left(\begin{array}{c}
n-1 \\
i
\end{array}\right)+\left(\begin{array}{c}
d \\
n-d
\end{array}\right)+\left(\begin{array}{c}
d-1 \\
n-d-1
\end{array}\right) .
$$

Many of the combinatorial properties of arrangements of (pseudo) hyperplanes can be studied in the language of oriented matroids. Indeed, an oriented matroid on $n$ elements of rank $r$ is naturally associated with every arrangement of $n$ (pseudo) hyperplanes in dimension $d=r-1$, and conversely the oriented matroids (without loops or parallel elements) are precisely those associated with some arrangement of pseudo hyperplanes, see [3]. In particular, cyclic arrangements of $n$ hyperplanes in $\mathbb{P}^{d}$ are equivalent to alternating oriented matroids of rank $r=d+1$ on $n$ elements.

By using this combinatorial description, we may reformulate (1) as follows.

$$
f(r, n)=\sum_{i=0}^{r-3}\left(\begin{array}{c}
n-1 \\
i
\end{array}\right)+\left(\begin{array}{c}
r-1 \\
n-r+1
\end{array}\right)+\left(\begin{array}{c}
r-2 \\
n-r
\end{array}\right) .
$$

In view of Roudneff's conjecture, we pose the following

Question 2. Is it true that every arrangement with $n \geqslant r \geqslant 3$ hyperplanes in $\mathbb{P}^{r-1}$ has at most $f(r, n)$ complete cells ? 
Notice that there is a unique arrangement of 3 (resp. 4) lines with $f(3,3)=4$ (resp. $f(3,4)=3)$ complete cells. Since Conjecture 1 is true for $r=3$ for any $n \geqslant 5$ then we have that Question 2 holds for $r=3$ and any $n \geqslant 3$.

In this note we answer positively Question 2 for arrangements of hyperplanes arising from Lawrence oriented matroids.

Theorem 3. Any arrangement with $n \geqslant r \geqslant 3$ hyperplanes in $\mathbb{P}^{r-1}$ arising from an acyclic Lawrence oriented matroid has at most $f(r, n)$ complete cells.

Notice that Theorem 3 implies Roudneff's conjecture when $n \geqslant 2 d+1 \geqslant 5$ for arrangements arising from an infinite family of Lawrence oriented matroids and thus giving more credit to the general conjecture. As we will see below, the class of Lawrence arrangements contains as a very particular case all the cyclic arrangements and thus a natural class to investigate the validity of the above question.

\section{Lawrence Oriented Matroids}

Recall that an oriented matroid $\mathcal{M}$ is acyclic if it does not contain positive circuits (otherwise, $\mathcal{M}$ is called cyclic). We say that an element $e \in E$ of an oriented acyclic matroid is interior if there exists a signed circuit $C=\left(C^{+}, C^{-}\right)$with $C^{-}=\{e\}$. It is well known that the cells (respectively complete cells) of a hyperplane arrangement are in oneto-one correspondence with the acyclic reorientations (respectively acyclic reorientations without interior elements) of the corresponding oriented matroid (see $[1,3,5]$ for more general results). We will use this combinatorial description to prove Theorem 3 .

A Lawrence oriented matroid $\mathcal{M}$ of rank $r$ on the totally ordered set $E=\{1, \ldots, n\}$, $r \leqslant n$, is a uniform oriented matroid obtained as the union of $r$ uniform oriented matroids $\mathcal{M}_{1}, \ldots, \mathcal{M}_{r}$ of rank 1 on $(E,<)$ (see $\left.[6,11]\right)$.

We can also define the Lawrence oriented matroids via the signature of their bases, that is via their chirotope $\chi$. Indeed, the chirotope $\chi$ corresponds to some Lawrence oriented matroid $\mathcal{M}_{A}$ if and only if there exists a matrix $A=\left(a_{i, j}\right), 1 \leqslant i \leqslant r, 1 \leqslant j \leqslant n$ with entries from $\{+1,-1\}$ (where the $i$ th row corresponds to the chirotope of the oriented matroid $\mathcal{M}_{i}$ ) such that

$$
\chi(B)=\prod_{i=1}^{r} a_{i, j_{i}}
$$

where $B$ is an ordered $r$-tuple $j_{1} \leqslant \ldots \leqslant j_{r}$ elements of $E$.

Remark 4. Let $A=\left(a_{i, j}\right), 1 \leqslant i \leqslant r, 1 \leqslant j \leqslant n$ be a matrix with entries from $\{+1,-1\}$ and $\mathcal{M}_{A}$ its corresponding Lawrence oriented matroid.

(i) The coefficients $a_{i, j}$ with $i>j$ or $j-n>i-r$ do not play any role in the definition of $\mathcal{M}_{A}$ (since they never appear in (3)). So, we may give them any arbitrary value from $\{+1,-1\}$ or ignore them completely. 
(ii) An opposite chirotope $-\chi$ is obtained by reversing the sign of all the coefficients of a line of $A$.

(iii) The oriented matroid ${ }_{\bar{c}} \mathcal{M}_{A}$ is obtained by reversing the sign of all the coefficients of a column $c$ in $A$.

(iv) If $a_{i, j}=1$ for all $i>j$ or $j-n>i-r$ then $\mathcal{M}_{A}$ is the alternating oriented matroid.

Let $\mathcal{M}_{A}$ be a Lawrence oriented matroid and $A$ the matrix associated $A=\left(a_{i, j}\right)$ with $1 \leqslant i \leqslant r, 1 \leqslant j \leqslant n$ and entries from $\{+1,-1\}$. In [9] were constructed the Top Travel $[T T]$ (and the Bottom Travel $[B T]$ ) on the entries of $A$, formed by horizontal and vertical movements according to the following procedure, illustrated in Figure 1.

\section{Procedure}

(1) $T T(B T)$ starts at $a_{1,1}\left(\right.$ at $\left.a_{r, n}\right)$

(2) Suppose that $T T(B T)$ arrives at $a_{i, j}$. Let $s\left(s^{\prime}\right)$ be the minimum (maximal) integer $j<s \leqslant n\left(1 \leqslant s^{\prime}<j\right)$ such that $a_{i, j}=-a_{i, s}\left(a_{i, j}=-a_{i, s^{\prime}}\right)$.

(3) If $s\left(s^{\prime}\right)$ does not exists then $T T$ goes horizontally to $a_{i, n}$ and stops (BT goes horizontally to $a_{i, 1}$ and stops)

(4) else

(a) if $1 \leqslant i \leqslant r-1(2 \leqslant i \leqslant r)$ then

$T T$ goes horizontally to $a_{i, s}$ and then goes vertically to $a_{i+1, s}$

(BT goes horizontally to $a_{i, s^{\prime}}$ and then goes vertically to $a_{i-1, s^{\prime}}$ )

(a) else

$T T$ goes horizontally to $a_{r, s}$ and stops

(BT goes horizontally to $a_{1, s^{\prime}}$ and stops)

\begin{tabular}{|c|c|c|c|c|c|c|c|}
\hline 1 & 2 & 3 & 4 & 5 & 6 & 7 \\
\hline \multirow{2}{*}{1} & - & - & + & + & + & + \\
\hline & + & - & + & + & + & + & + \\
\hline 3 & + & + & + & + & + & + & + \\
\hline & + & & & & & & \\
\hline & + & + & + & + & + & + & + \\
\hline
\end{tabular}

Figure 1: Matrix $A^{\prime}$ with corresponding Top and Bottom Travels. 
We write $T T=\left(a_{1,1}, \ldots, a_{1, t_{1}}, a_{2, t_{1}}, \ldots, a_{2, t_{2}}, \ldots, a_{x, t_{x-1}}, \ldots, a_{x, t_{x}}\right), 1 \leqslant x \leqslant r+1$, where $a_{l, t_{l-1}}, \ldots, a_{l, t_{l}}$ are the entries in line $l$ of $A$ along $T T$ with $1 \leqslant l \leqslant x \leqslant r+1$. Let $A=\left(a_{i, j}\right), 1 \leqslant i \leqslant r, 1 \leqslant j \leqslant n$ with entries from $\{+1,-1\}$. We define a plain travel $P$ on the entries of $A$, formed by horizontal (from left to right) and vertical (from top to bottom) movements such that

(a) $P$ starts with $a_{1,1}, a_{1,2}$

(b) $P$ cannot make two consecutive vertical movements and

(c) $P$ ends at $a_{i, n}$ for $1 \leqslant i \leqslant r$.

In $[9$, Lemma 3.1] was given a bijection between the set of all plain travels of $A$ and the set of all acyclic reorientations of $\mathcal{M}_{A}$ given as follows : associate to $P$ the set of elements of $\mathcal{M}_{A}$ that should be reoriented to transform $P$ to the Top Travel of the new matrix $A^{P}=\left(a_{i, j}^{P}\right)$ (obtained by reversing the signs of all coefficients of the columns in $A$ corresponding the reoriented elements). Let us denote by $P^{-}$the Bottom Travel in $A^{P}$ and call it the inverse plain travel of $P$ in $A$. Notice that $P^{-}$also have similar properties as those defined for $P$, that is, (a) $P^{-}$starts with $a_{r, n}, a_{r, n-1},(b) P^{-}$cannot make two consecutive vertical movements and $(c) P^{-}$ends at $a_{i, 1}$ for $1 \leqslant i \leqslant r$.

Remark 5. Similarly as in [9, Lemma 3.1] it can be proved that there is a bijection between all acyclic orientations of $\mathcal{M}_{A}$ and the set of all inverse plain travels in $A$, and thus, there is a bijection between the set of all plain travels and the set of all inverse plain travels.

The following two lemmas allow us to detect when $\mathcal{M}_{A}$ is acyclic and when it has interior elements (if any) in terms of $T T$ and $B T$.

Lemma 6. [9] Let $\mathcal{M}_{A}$ be a Lawrence oriented matroid and $A$ the matrix associated $A=\left(a_{i, j}\right)$ with $1 \leqslant i \leqslant r, 1 \leqslant j \leqslant n$ and entries from $\{+1,-1\}$. Then the following conditions are equivalent.

(a) $\mathcal{M}_{A}$ is cyclic,

(b) $T T$ ends at $a_{r, s}$ for some $1 \leqslant s<n$,

(c) BT ends at $a_{1, s^{\prime}}$ for some $1<s \leqslant n$.

We say that $T T$ and $B T$ are parallel at column $k$ with $2 \leqslant k \leqslant n-1$ in $A$ if $T T=\left(a_{1,1}, \ldots, a_{i, k-1}, a_{i, k}, a_{i, k+1}, \ldots\right)$ and either $B T=\left(a_{r, n}, \ldots, a_{i, k+1}, a_{i, k}, a_{i, k-1}, \ldots\right)$ or $B T=\left(a_{r, n}, \ldots, a_{i+1, k+1}, a_{i+1, k}, a_{i+1, k-1}, \ldots\right), 1 \leqslant i \leqslant r$.

Lemma 7. [9] Let $\mathcal{M}_{A}$ be a Lawrence oriented matroid and $A$ the matrix associated $A=\left(a_{i, j}\right)$ with $1 \leqslant i \leqslant r, 1 \leqslant j \leqslant n$ and entries from $\{+1,-1\}$. Then $k$ is an interior element of $\mathcal{M}_{A}$ if and only if

(a) $B T=\left(a_{r, n}, \ldots, a_{1,2}, a_{1,1}\right)$ for $k=1$,

(b) $T T=\left(a_{1,1}, \ldots, a_{r, n-1}, a_{r, n}\right)$ for $k=n$, 
(c) TT and BT are parallel at $k$ for $2 \leqslant k \leqslant n-1$.

Example. Let $M_{A^{\prime}}$ be the Lawrence oriented matroid associated to the matrix $A^{\prime}$ given in Figure 1. We notice that $M_{A^{\prime}}$ is acyclic and that 4,5 and 6 are interior elements, see Figure 1.

\section{Main result}

Throughout the rest of the paper only acyclic Lawrence oriented matroids $\mathcal{M}_{A}$ will be considered. Let $g_{A}(r, n)$ be the number of complete cells of the arrangements obtained from the acyclic Lawrence oriented matroid $\mathcal{M}_{A}$ of rank $r$ on $n$ elements. We thus have that $g_{A}(r, n)$ is the number of plain travels in $A$ without interior elements.

Our main aim is to show that

$$
g_{A}(r, n) \leqslant f(r, n)
$$

for every matrix $A=\left(a_{i, j}\right), 1 \leqslant i \leqslant r, 1 \leqslant j \leqslant n, n \geqslant r \geqslant 3$ with entries from $\{+1,-1\}$.

Let $B T_{r \times n}^{t}$ be the set of all inverse travels $P^{-}$of a $(r \times n)$-matrix not arriving at line $t$ with $0 \leqslant t \leqslant r-1$. (See Figure 2.)

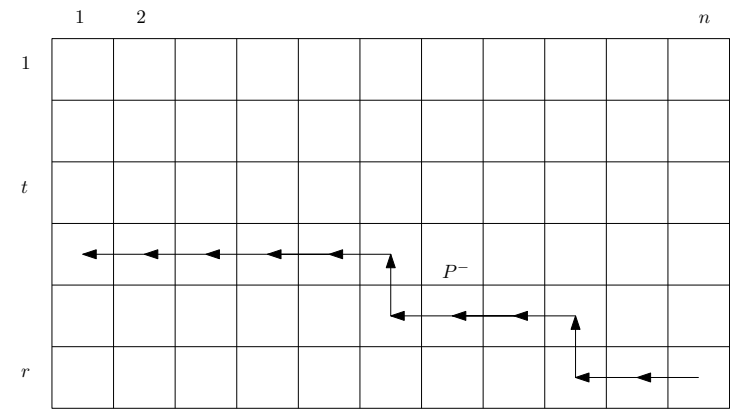

Figure 2: An inverse travel $P^{-} \in B T_{r \times n}^{t}$.

Remark 8. For every integers $t, r, n$ with $0 \leqslant t \leqslant r-1, n \leqslant r$ we have

$$
\left|B T_{r \times n}^{t}\right|=\sum_{i=0}^{r-t-1}\left(\begin{array}{c}
n-1 \\
i
\end{array}\right) .
$$

Proof. Let $A$ be a $(r \times n)$-matrix. By Remark 5 the set of all inverse plain travels in $\mathcal{M}_{A}$ are in bijection with the set of all acyclic reorientations, wich correspond to plain travels in $A$, and thus equals to $\sum_{i=0}^{r-1}\left(\begin{array}{c}n-1 \\ i\end{array}\right)$ (see [9, Section 3]). Since $\left|B T_{r \times n}^{t}\right|$ is exactly the number of all plain travels in a matrix with $r-t$ lines and $n$ columns, then the equality follows. 
Let $P T_{j}^{A}, 2 \leqslant j \leqslant n$ be the set of all plain travels $P$ in $A$ without interior elements and such that $a_{1, j}, a_{2, j} \in P$ (that is, travels in which the first vertical movement is done at column $j$, see Figure 3 ). We also let $P T_{n+1}^{A}$ the travel $a_{1,1}=\cdots=a_{1, n}$ without interior elements (this might not exist). We immediately have that

$$
g_{A}(r, n)=\sum_{j=2}^{n+1}\left|P T_{j}^{A}\right| .
$$

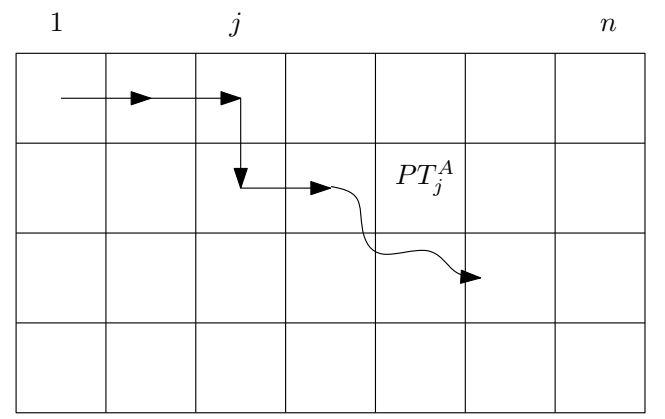

Figure 3: The set of plain travels $P T_{j}^{A}$.

We may now intend to give suitable upper bounds to the cardinality of $P T_{j}^{A}$ for any $j$ (Propositions 10 and 12). The latter will be done by using two (short) lemmas (Lemmas 9 and 11).

Lemma 9. Let $A=\left(a_{i, j}\right), 1 \leqslant i \leqslant r, 1 \leqslant j \leqslant n$ with entries from $\{+1,-1\}$ and $n \geqslant r \geqslant 3$. Let $P \in P T_{j}^{A}$, with $3 \leqslant j \leqslant n+1$, then $P^{-}$does not arrive at line 2 in column $s$, for every $s \geqslant 3$.

Proof. By definition of $P T_{j}^{A}$, we have that $a_{1, k} \in P$ for every $k=1, \ldots, j$. Suppose by contradiction that $P^{-}$arrives at $a_{2, s}$ for some $s \geqslant 3$, then by Lemma 7 , one can easily check that one of the elements corresponding to columns $1 \leqslant t \leqslant s-1$ would be an interior element, contradicting the hypothesis that $P$ has not interior elements.

We notice that we need $j>2$, otherwise we can have a $P \in P T_{2}^{A}$ with $P^{-}$arriving at line 2 at column $s$ (Figure 4 illustrates this with $s=3$ ). Figure 6 shows that it is also necessary to have $s \geqslant 3$.

Given a matrix $A=\left(a_{i, j}\right), 1 \leqslant i \leqslant r, 1 \leqslant j \leqslant n$ with entries from $\{+1,-1\}$, we define the submatrix $A_{j}:=\left(a_{i, k}\right)$ with $2 \leqslant i \leqslant r$ and $j \leqslant k \leqslant n$, see Figure 5 .

Proposition 10. Let $A=\left(a_{i, j}\right), 1 \leqslant i \leqslant r, 1 \leqslant j \leqslant n$ with entries from $\{+1,-1\}$ and $n \geqslant r \geqslant 3$. Then,

(a) $\left|P T_{j}^{A}\right| \leqslant g_{A_{j}}(r-1, n-j+1)$ for every $j=2, \ldots, n$, 


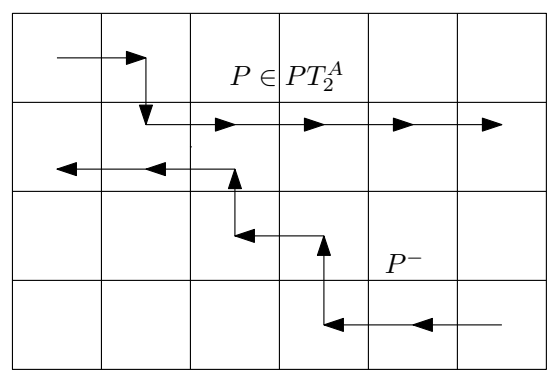

Figure 4: An example showing $P \in P T_{2}^{A}$ and $P^{-}$arriving at line 2 .

(b) $\left|P T_{j}^{A}\right| \leqslant \sum_{i=0}^{r-3}\left(\begin{array}{c}n-j \\ i\end{array}\right)$ for every $j=3, \ldots, n$.

Proof. (a) By definition, the submatrix $A_{j}$ cannot have more than $g_{A_{j}}(r-1, n-j+1)$ plain travels without interior elements. Now, since for each $P \in P T_{j}^{A}$ we have $a_{2, j}, a_{2, j+1} \in P$, it follows that $P$ restricted to the submatrix $A_{j}$ is a plain travel in $A_{j}$ without interior element and thus $\left|P T_{j}^{A}\right| \leqslant g_{A_{j}}(r-1, n-j+1)$.

(b) By Lemma 9 for every $j=3, \ldots, n$ and every $P \in P T_{j}^{A}$ we have that $P^{-}$does not arrive at line 2 in column $s$ for every $s \geqslant 3$. Therefore, the inverse plain travel $P^{-}$, restricted to $A_{j}$, is an inverse plain travel in $B T_{(r-1) \times(n-j+1)}^{1}$ (see Figure 5). Since there is a bijection between the set of plain travels and the set of inverse plain travels, we have $\left|P T_{j}^{A}\right| \leqslant\left|B T_{(r-1) \times(n-j+1)}^{1}\right|$ for every $j=3, \ldots, n$. The result follows by Remark 8 .

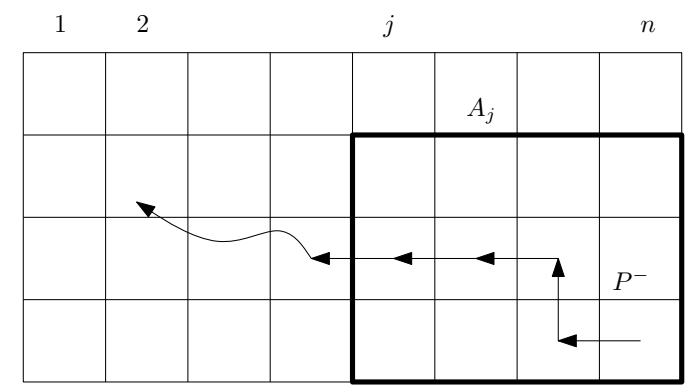

Figure 5: $P^{-}$, restricted to $A_{j}$, is an inverse plain travel in $B T_{(r-1) \times(n-j+1)}^{1}$.

Example (continuation). By Proposition 10 (a) we have that $\left|P T_{j}^{A^{\prime}}\right| \leqslant g_{A_{j}^{\prime}}(3,8-j)$ for every $j=2, \ldots, 7$ and by Proposition $10(b)$ we have that $\left|P T_{j}^{A^{\prime}}\right| \leqslant 8-j$ for every $j=3, \ldots, 7$. For instance, if $j=5$, the submatrix $A_{5}^{\prime}$ of $A^{\prime}$ induce the alternating oriented matroid of rank 3 with 3 elements, and so by $(a)$, we obtain $\left|P T_{5}^{A^{\prime}}\right| \leqslant g_{A_{5}^{\prime}}(3,3)=f(3,3)=$ 4 while by $(b)$ we have that $\left|P T_{5}^{A^{\prime}}\right| \leqslant 3$.

In order to improve Proposition 10 (b) when $j \geqslant 4$, we need the following lemma. 
Lemma 11. Let $A=\left(a_{i, j}\right), 1 \leqslant i \leqslant r, 1 \leqslant j \leqslant n$ with entries from $\{+1,-1\}$ and $n \geqslant r \geqslant 4$. Let $P_{1} \in P T_{j}^{A}$, with $4 \leqslant j \leqslant n$ corresponding to the Top Travel of the matrix $A^{P_{1}}=\left(a_{i, j}^{P_{1}}\right)$ and suppose that $P_{1}^{-}$arrive at line 3 in column $s$ for some $s \geqslant j$. Then $a_{3, j-1}^{P_{1}}=a_{3, j}^{P_{1}}$.

Moreover, for each $k \in\{j+1, \ldots, n+1\}$ and each $P_{2} \in P T_{k}^{A}$ we have that $P_{2}^{-}$does not arrive at line 3 in column $t$ for every $t \geqslant j$.

Proof. Let $P_{1} \in P T_{j}^{A}$, with $4 \leqslant j \leqslant n$. Since $P_{1}^{-}$arrive at line 3 in column $s$ for some $s \geqslant j$, it follows by Lemma 9 , that $a_{3,3}^{P_{1}}=a_{3,4}^{P_{1}}=\cdots=a_{3, s}^{P_{1}}$, in particular $a_{3, j-1}^{P_{1}}=a_{3, j}^{P_{1}}$.

Let $k \in\{j+1, \ldots, n+1\}$ and $P_{2} \in P T_{k}^{A}$ (corresponding to the Top Travel of the matrix $\left.A^{P_{2}}=\left(a_{i, j}^{P_{2}}\right)\right)$ and suppose, by contradiction, that there exists $t \geqslant j$ such that $P_{2}^{-}$arrive at line 3 in column $t$. By Lemma 9 we have that $a_{3,3}^{P_{2}}=a_{3,4}^{P_{2}}=\cdots=a_{3, t}^{P_{2}}$, in particular, $a_{3, j-1}^{P_{2}}=a_{3, j}^{P_{2}}$. Since $a_{1, j-1}^{P_{1}} \neq a_{1, j}^{P_{1}}$ and $a_{1, j-1}^{P_{2}}=a_{1, j}^{P_{2}}$ we have that the acyclic reorientation in $A^{P_{1}}$ that yields to $A^{P_{2}}$ (i.e., the acyclic reorientation in $A^{P_{1}}$ which makes $P_{2}$ Top Travel) has to reorient only one of the columns $j-1$ and $j$ in order to have $a_{1, j-1}^{P_{2}}=a_{1, j}^{P_{2}}$. Hence, as $a_{3, j-1}^{P_{1}}=a_{3, j}^{P_{1}}$ it follows that $a_{3, j-1}^{P_{2}} \neq a_{3, j}^{P_{2}}$ which is a contradiction.

Figure 6 shows that Lemma 11 is not true for $j=3$.

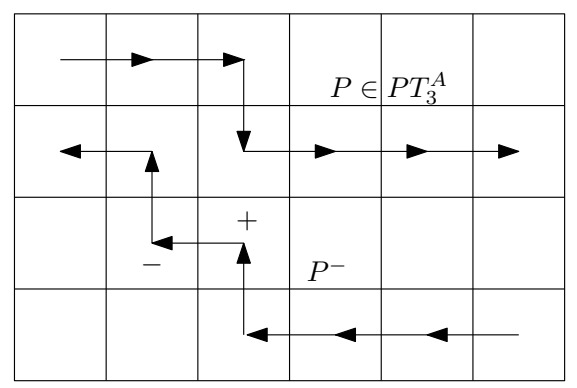

Figure 6: An example of $P \in P T_{3}^{A}$ with $P^{-}$arriving at line 3 in column 3 with $a_{3,2}^{P} \neq a_{3,3}^{P}$.

Proposition 12. Let $A=\left(a_{i, j}\right), 1 \leqslant i \leqslant r, 1 \leqslant j \leqslant n$ with entries from $\{+1,-1\}$ and $n \geqslant r \geqslant 4$. Let $s$ be the smallest integer in $\{4, \ldots, n-1\}$ such that $\left|P T_{s}^{A}\right|>\sum_{i=0}^{r-4}\left(\begin{array}{c}n-s \\ i\end{array}\right)$. Then,

$$
\sum_{k=s+1}^{n+1}\left|P T_{k}^{A}\right| \leqslant \sum_{i=0}^{r-4}\left(\begin{array}{c}
n-s \\
i
\end{array}\right) .
$$

If such $s$ does not exist, then

$$
\left|P T_{j}^{A}\right| \leqslant \sum_{i=0}^{r-4}\left(\begin{array}{c}
n-j \\
i
\end{array}\right)
$$

for every $j=4, \ldots, n$. 
Proof. Since $\left|P T_{s}^{A}\right|>\sum_{i=0}^{r-4}\left(\begin{array}{c}n-s \\ i\end{array}\right)=\left|B T_{r \times(n-s+1)}^{3}\right|$, then there exists $P_{1} \in P T_{s}^{A}$ such that $P_{1}^{-}$arrives at line 3 in column $m$ for some $m \geqslant s$. Thus, by Lemma 11 for every $k \in\{s+1, \ldots, n+1\}$ and $P_{2} \in P T_{k}^{A}$, we have that $P_{2}^{-}$does not arrive at line 3 in column $t$ for every $t \geqslant s$, that is to say, $P_{2} \in B T_{r \times(n-s+1)}^{3}$. Hence, $\sum_{k=s+1}^{n+1}\left|P T_{k}^{A}\right| \leqslant\left|B T_{r \times(n-s+1)}^{3}\right|=$ $\sum_{i=0}^{r-4}\left(\begin{array}{c}n-s \\ i\end{array}\right)$. If such $s$ does not exist then clearly $\left|P T_{j}^{A}\right| \leqslant \sum_{i=0}^{r-4}\left(\begin{array}{c}n-j \\ i\end{array}\right)$ for every $j=4, \ldots, n-1$. Finally, since $\left|P T_{n}^{A}\right| \leqslant 1$ and $\sum_{i=0}^{r-4}\left(\begin{array}{c}n-n \\ i\end{array}\right)=1$, then $\left|P T_{j}^{A}\right| \leqslant \sum_{i=0}^{r-4}\left(\begin{array}{c}n-j \\ i\end{array}\right)$ for $j=n$.

Example (continuation). The Top Travel $P_{1}$ corresponding to reverse the sign of all the coefficients of column 1 of the matrix $A^{\prime}=\left(a_{i, j}^{\prime}\right), 1 \leqslant i \leqslant 4,1 \leqslant j \leqslant 7$ given in Figure 1, has no interior elements. Since $a_{1,4}^{\prime}, a_{2,4}^{\prime} \in P_{1}$, the plane travel $P_{1}$ of $A^{\prime}$ is in $P T_{4}^{A^{\prime}}$. Simmilarly, the Top Travel $P_{2}$ corresponding to reverse the sign of all the coefficients of columns $2,3,4$ and 5 of $A^{\prime}$ has no interior elements. Since $a_{1,4}^{\prime}, a_{2,4}^{\prime} \in P_{2}$, it follows that $P_{2} \in P T_{4}^{A^{\prime}}$. Hence $\left|P T_{4}^{A^{\prime}}\right|>\sum_{i=0}^{r-4}\left(\begin{array}{c}n-s \\ i\end{array}\right)=\sum_{i=0}^{0}\left(\begin{array}{c}7-4 \\ i\end{array}\right)=1$. So, by Proposition 12 we obtain that $\sum_{k=5}^{8}\left|P T_{k}^{A^{\prime}}\right| \leqslant \sum_{i=0}^{r-4}\left(\begin{array}{c}n-s \\ i\end{array}\right)=1$. Furthermore, it is not difficult to check that $\left|P T_{2}^{A^{\prime}}\right|=\left|P T_{3}^{A^{\prime}}\right|=1$ and $\left|P T_{2}^{A^{\prime}}\right|=2$ obtaining that $g_{A^{\prime}}(3,4)=\sum_{i=2}^{8}\left|P T_{i}^{A^{\prime}}\right| \leqslant 5$.

Now, we may use Propositions 10 and 12 to give an upper bound of $g_{A}(r, n)$.

Lemma 13. Let $A=\left(a_{i, j}\right), 1 \leqslant i \leqslant r, 1 \leqslant j \leqslant n$ with entries from $\{+1,-1\}$ with $n \geqslant r \geqslant 4$. Then, we have either

$$
\begin{aligned}
g_{A}(r, n) & \leqslant g_{A_{2}}(r-1, n-1)+\min \left\{\sum_{i=0}^{r-3}\left(\begin{array}{c}
n-3 \\
i
\end{array}\right), g_{A_{3}}(r-1, n-2)\right\} \\
& +\sum_{j=4}^{n} \min \left\{\sum_{i=0}^{r-4}\left(\begin{array}{c}
n-j \\
i
\end{array}\right), g_{A_{j}}(r-1, n-j+1)\right\}+1:=\mu_{g_{A}}(r, n),
\end{aligned}
$$

or there exists $s \in\{4 \ldots, n-1\}$ such that

$$
\begin{aligned}
g_{A}(r, n) & \leqslant g_{A_{2}}(r-1, n-1)+\min \left\{\sum_{i=0}^{r-3}\left(\begin{array}{c}
n-3 \\
i
\end{array}\right), g_{A_{3}}(r-1, n-2)\right\} \\
& +\sum_{j=4}^{s-1} \min \left\{\sum_{i=0}^{r-4}\left(\begin{array}{c}
n-j \\
i
\end{array}\right), g_{A_{j}}(r-1, n-j+1)\right\} \\
& +\min \left\{\sum_{i=0}^{r-3}\left(\begin{array}{c}
n-s \\
i
\end{array}\right), g_{A_{s}}(r-1, n-s+1)\right\}+\sum_{i=0}^{r-4}\left(\begin{array}{c}
n-s \\
i
\end{array}\right):=\lambda_{g_{A}}(r, n, s) .
\end{aligned}
$$


Proof. First suppose that there is not an integer $s \in\{4, \ldots, n-1\}$ such that $\left|P T_{s}^{A}\right|>$ $\sum_{i=0}^{r-4}\left(\begin{array}{c}n-s \\ i\end{array}\right)$. Then, by Proposition 12 , we have that $\sum_{j=4}^{n}\left|P T_{j}^{A}\right| \leqslant \sum_{i=0}^{r-4}\left(\begin{array}{c}n-j \\ i\end{array}\right)$. By Proposition 10 we have that $\left|P T_{j}^{A}\right| \leqslant g_{A}(r-1, n-j+1)$ for $j=2,3$ and $\left|P T_{3}^{A}\right| \leqslant \sum_{i=0}^{r-3}\left(\begin{array}{c}n-3 \\ i\end{array}\right)$. Therefore, we have that $\sum_{j=2}^{n}\left|P T_{j}^{A}\right| \leqslant \mu_{g_{A}}(r, n)-1$. Since $\left|P T_{n+1}^{A}\right| \leqslant 1$ and $g_{A}(r, n)=\sum_{j=2}^{n+1}\left|P T_{j}^{A}\right|$, it easily follows that $g_{A}(r, n) \leqslant \mu_{g_{A}}(r, n)$.

Now, suppose that there exists $s \in\{4, \ldots, n-1\}$ such that $\left|P T_{s}^{A}\right|>\sum_{i=0}^{r-4}\left(\begin{array}{c}n-s \\ i\end{array}\right)$. We may suppose that $s$ is the smallest integer with this property, that is if $s>4$ then $\left|P T_{j}^{A}\right| \leqslant \sum_{i=0}^{r-4}\left(\begin{array}{c}n-j \\ i\end{array}\right)$ for any $j \in\{4, \ldots, s-1\}$. Then, by Proposition 12, we have that $\sum_{k=s+1}^{n+1}\left|P T_{k}^{A}\right| \leqslant \sum_{i=0}^{r-4}\left(\begin{array}{c}n-s \\ i\end{array}\right)$. Hence, by the latter and by Proposition 10, it can be easily deduced that $g_{A}(r, n)=\sum_{j=2}^{n+1}\left|P T_{j}^{A}\right| \leqslant \lambda_{g_{A}}(r, n, s)$.

Example (continuation). We can now compute $\mu_{g_{A^{\prime}}}(4,7)$ and $\lambda_{g_{A^{\prime}}}(4,7, s)$ in order to obtain an upper bound for $g_{A^{\prime}}(4,7)$. It is not difficult to check that $g_{A_{2}^{\prime}}(3,6)=1$, $g_{A_{3}^{\prime}}(3,5)=1, g_{A_{4}^{\prime}}(3,4)=f(3,4)=3, g_{A_{5}^{\prime}}(3,3)=f(3,3)=4, g_{A_{6}^{\prime}}(3,2)=f(3,2)=2$ and $g_{A_{7}^{\prime}}(3,1)=f(3,1)=1$. Then, one can check that $\mu_{g_{A^{\prime}}}(4,7)=7, \lambda_{g_{A^{\prime}}}(4,7,4)=6$, $\lambda_{g_{A^{\prime}}}(4,7,5)=7$ and $\lambda_{g_{A^{\prime}}}(4,7,6)=7$.

Let us define $g(r, n)=f(r, n)=0$ if $n<r$. We will now define two parameters, $\mu_{f}(r, n)$ and $\lambda_{f}(r, n, s)$, that will be used to give upper bounds for $\mu_{g_{A}}(r, n)$ and $\lambda_{g_{A}}(r, n, s)$ respectively. The latter will imply the desired upper bound for $g_{A}(r, n)$ since $\lambda_{f}(r, n, s) \leqslant$ $\mu_{f}(r, n)$ (Lemma 14) and, in fact, $\mu_{f}(r, n)=f(r, n)$ (see proof of Theorem 3).

For every integers $n \geqslant r \geqslant 4$, we define

$$
\begin{aligned}
\mu_{f}(r, n):=f( & r-1, n-1)+\min \left\{\sum_{i=0}^{r-3}\left(\begin{array}{c}
n-3 \\
i
\end{array}\right), f(r-1, n-2)\right\} \\
& +\sum_{j=4}^{n} \min \left\{\sum_{i=0}^{r-4}\left(\begin{array}{c}
n-j \\
i
\end{array}\right), f(r-1, n-j+1)\right\}+1
\end{aligned}
$$

and for every $s \in\{4, \ldots, n-1\}$, we define

$$
\begin{aligned}
\lambda_{f}(r, n, s):=f( & r-1, n-1)+\min \left\{\sum_{i=0}^{r-3}\left(\begin{array}{c}
n-3 \\
i
\end{array}\right), f(r-1, n-2)\right\} \\
& +\sum_{j=4}^{s-1} \min \left\{\sum_{i=0}^{r-4}\left(\begin{array}{c}
n-j \\
i
\end{array}\right), f(r-1, n-j+1)\right\}
\end{aligned}
$$




$$
+\min \left\{\sum_{i=0}^{r-3}\left(\begin{array}{c}
n-s \\
i
\end{array}\right), f(r-1, n-s+1)\right\}+\sum_{i=0}^{r-4}\left(\begin{array}{c}
n-s \\
i
\end{array}\right) .
$$

By equality (2), we may replace the values of $f(r-1, n-j)$, for every $j=1, \ldots, n-1$, in (7), obtaining

$$
\begin{aligned}
& \mu_{f}(r, n) \\
& =\sum_{i=0}^{r-4}\left(\begin{array}{c}
n-2 \\
i
\end{array}\right)+\min \left\{\sum_{i=0}^{r-3}\left(\begin{array}{c}
n-3 \\
i
\end{array}\right), \sum_{i=0}^{r-4}\left(\begin{array}{c}
n-3 \\
i
\end{array}\right)\right\} \\
& +\sum_{j=4}^{n} \min \left\{\sum_{i=0}^{r-4}\left(\begin{array}{c}
n-j \\
i
\end{array}\right), \sum_{i=0}^{r-4}\left(\begin{array}{c}
n-j \\
i
\end{array}\right)+\left(\begin{array}{c}
r-2 \\
n-j-r+3
\end{array}\right)+\left(\begin{array}{c}
r-3 \\
n-j-r+2
\end{array}\right)\right\}+1 .
\end{aligned}
$$

Hence, $\mu_{f}(r, n)=\sum_{i=0}^{r-4}\left(\begin{array}{c}n-2 \\ i\end{array}\right)+\sum_{i=0}^{r-4}\left(\begin{array}{c}n-3 \\ i\end{array}\right)+\sum_{j=4}^{n} \sum_{i=0}^{r-4}\left(\begin{array}{c}n-j \\ i\end{array}\right)+1$ and so,

$$
\mu_{f}(r, n)=\sum_{j=2}^{n} \sum_{i=0}^{r-4}\left(\begin{array}{c}
n-j \\
i
\end{array}\right)+1 .
$$

Similarly, by equality (2), we may replace the values of $f(r-1, n-j)$, for every $j=$ $1, \ldots, s-1$, in (10), obtaining

$$
\begin{aligned}
\lambda_{f}(r, n, s) & =\sum_{i=0}^{r-4}\left(\begin{array}{c}
n-2 \\
i
\end{array}\right)+\sum_{i=0}^{r-4}\left(\begin{array}{c}
n-3 \\
i
\end{array}\right)+\sum_{j=4}^{s-1} \sum_{i=0}^{r-4}\left(\begin{array}{c}
n-j \\
i
\end{array}\right)+\sum_{i=0}^{r-4}\left(\begin{array}{c}
n-s \\
i
\end{array}\right) \\
& +\min \left\{\sum_{i=0}^{r-3}\left(\begin{array}{c}
n-s \\
i
\end{array}\right), \sum_{i=0}^{r-4}\left(\begin{array}{c}
n-s \\
i
\end{array}\right)+\left(\begin{array}{c}
r-2 \\
n-s-r+3
\end{array}\right)+\left(\begin{array}{c}
r-3 \\
n-s-r+2
\end{array}\right)\right\}
\end{aligned}
$$

Hence,

$$
\begin{aligned}
\lambda_{f}(r, n, s) & =\sum_{j=2}^{s} \sum_{i=0}^{r-4}\left(\begin{array}{c}
n-j \\
i
\end{array}\right) \\
& +\min \left\{\sum_{i=0}^{r-3}\left(\begin{array}{c}
n-s \\
i
\end{array}\right), \sum_{i=0}^{r-4}\left(\begin{array}{c}
n-s \\
i
\end{array}\right)+\left(\begin{array}{c}
r-2 \\
n-s-r+3
\end{array}\right)+\left(\begin{array}{c}
r-3 \\
n-s-r+2
\end{array}\right)\right\} .
\end{aligned}
$$

Lemma 14. For every integers $r, n, s$ such that $n \geqslant r \geqslant 4$ and $n-1 \geqslant s \geqslant 4$, we have that $\mu_{f}(r, n) \geqslant \lambda_{f}(r, n, s)$.

Proof. By (11) and (12) we have that

$$
\mu_{f}(r, n)-\lambda_{f}(r, n, s)=\sum_{j=s+1}^{n} \sum_{i=0}^{r-4}\left(\begin{array}{c}
n-j \\
i
\end{array}\right)+1
$$




$$
-\min \left\{\sum_{i=0}^{r-3}\left(\begin{array}{c}
n-s \\
i
\end{array}\right), \sum_{i=0}^{r-4}\left(\begin{array}{c}
n-s \\
i
\end{array}\right)+\left(\begin{array}{c}
r-2 \\
n-s-r+3
\end{array}\right)+\left(\begin{array}{c}
r-3 \\
n-s-r+2
\end{array}\right)\right\} .
$$

We will first prove that $\sum_{j=s+1}^{n} \sum_{i=0}^{r-4}\left(\begin{array}{c}n-j \\ i\end{array}\right)+1=\sum_{i=0}^{r-3}\left(\begin{array}{c}n-s \\ i\end{array}\right)$. By using the classical recursive formula $\left(\begin{array}{c}n-s \\ i\end{array}\right)=\left(\begin{array}{c}n-s-1 \\ i-1\end{array}\right)+\left(\begin{array}{c}n-s-1 \\ i\end{array}\right)$, we obtain that $\left(\begin{array}{c}n-s \\ i\end{array}\right)=\sum_{j=1}^{n-s-i+1}\left(\begin{array}{c}n-s-j \\ i-1\end{array}\right)$ for every $i \geqslant 1$, and thus

$$
\begin{aligned}
\sum_{i=0}^{r-3}\left(\begin{array}{c}
n-s \\
i
\end{array}\right) & =\sum_{i=1}^{r-3}\left(\begin{array}{c}
n-s \\
i
\end{array}\right)+1 \\
& =\sum_{i=1}^{r-3} \sum_{j=1}^{n-s-i+1}\left(\begin{array}{c}
n-s-j \\
i-1
\end{array}\right)+1 \\
& =\sum_{i=0}^{r-4} \sum_{j=1}^{n-s-i}\left(\begin{array}{c}
n-s-j \\
i
\end{array}\right)+1 .
\end{aligned}
$$

Therefore, if $\sum_{i=0}^{r-3}\left(\begin{array}{c}n-s \\ i\end{array}\right)=\min \left\{\sum_{i=0}^{r-3}\left(\begin{array}{c}n-s \\ i\end{array}\right), \sum_{i=0}^{r-4}\left(\begin{array}{c}n-s \\ i\end{array}\right)+\left(\begin{array}{c}r-2 \\ n-s-r+3\end{array}\right)+\left(\begin{array}{c}r-3 \\ n-s-r+2\end{array}\right)\right\}$ it follows that $\mu_{f}(r, n)=\lambda_{f}(r, n, s)$. Otherwise, $\mu_{f}(r, n)>\lambda_{f}(r, n, s)$.

Proof of Theorem 3. Let $A=\left(a_{i, j}\right), 1 \leqslant i \leqslant r, 1 \leqslant j \leqslant n$ with entries from $\{+1,-1\}$ and $n \geqslant r \geqslant 3$. We shall prove that $g_{A}(r, n) \leqslant f(r, n)$ by induction on $r$ for $n \geqslant r \geqslant 3$. As discussed in the introduction, the result holds for any simple arrangement of lines, that is when $r=3$, in particular, it holds for arrangements arising from Lawrence oriented matroids.

Suppose now that $g_{A}(r-1, n) \leqslant f(r-1, n)$ for every $n \geqslant r-1$. By comparing (term by term) the equalities (5) and (7) we obtain

$$
\mu_{g_{A}}(r, n) \leqslant \mu_{f}(r, n) .
$$

Also for every $s \in\{4, \ldots, n-1\}$, by comparing (term by term) the equalities (6) and (10) we obtain

$$
\lambda_{g_{A}}(r, n, s) \leqslant \lambda_{f}(r, n, s) .
$$

By Lemma 13, either $g_{A}(r, n) \leqslant \mu_{g_{A}}(r, n)$ for every $n \geqslant r \geqslant 4$ or there exists $s \in$ $\{4 \ldots, n-1\}$ such that $g_{A}(r, n) \leqslant \lambda_{g_{A}}(r, n, s)$. By inequalities (13) and (14) and Lemma 14 we have that $\max \left\{\mu_{g_{A}}(r, n), \lambda_{g_{A}}(r, n, s)\right\} \leqslant \mu_{f}(r, n)$ for every $n \geqslant r \geqslant 4$ and every $s \in\{4, \ldots, n-1\}$. Thus

$$
g_{A}(r, n) \leqslant \mu_{f}(r, n)
$$

for every integers $n \geqslant r \geqslant 4$. But

$$
f(r, n)=\sum_{i=1}^{r-3} \sum_{j=1}^{n-i}\left(\begin{array}{c}
n-1-j \\
i-1
\end{array}\right)+1
$$




$$
\begin{aligned}
& =\sum_{j=1}^{n-i-1} \sum_{i=0}^{r-4}\left(\begin{array}{c}
n-1-j \\
i
\end{array}\right)+1 \\
& =\sum_{j=2}^{n-i} \sum_{i=0}^{r-4}\left(\begin{array}{c}
n-j \\
i
\end{array}\right)+1 \stackrel{(11)}{=} \mu_{f}(r, n) .
\end{aligned}
$$

Therefore, $g_{A}(r, n) \leqslant f(r, n)$.

\section{References}

[1] B. Björner, M. Las Vergnas, B. Sturmfels, and N. White, G. Ziegler. Oriented Matroids. Encyclopedia of Mathematics and its Applications, 1993.

[2] C. Carathéodory. Über den Variabilitätsbereich des Koeffizienten von Potentzreihen die gegberne Wette nicht annehmen. Math. Ann., 64:95-115, 1904.

[3] J. Folkman and J. Lawrence. Oriented matroids. J. Comb. Theory Ser. B, 25:199236, 1978.

[4] D. Forge and J.L. Ramírez Alfonsín. On counting the $k$-face cells of cyclic arrangements. Europ. J. Combinatorics, 22:307-312, 2001.

[5] M. Las Vergnas. Convexity in oriented matroids. J. Comb. Theory Ser. B, 29:231243, 1980.

[6] J. Lawrence and L. Weinberg. Unions of oriented matroids. Linear Algebr. Appl., 41:183-200, 1981.

[7] P. McMullen. The maximal number of faces of a convex polytope. Mathematika, 17:179-184, 1970.

[8] J.L. Ramírez Alfonsín. Cyclic arrangements and Roudneff's conjecture in the space. Inform. Process. Lett., 71:179-182, 1999.

[9] J.L. Ramírez Alfonsín. Lawrence Oriented Matroids and a Problem of McMullen on Projective Equivalences of Polytopes. Europ. J. Combinatorics, 22:723-731, 2001.

[10] J.-P. Roudneff. Cells with many facets in arrangements of hyperplanes. Discrete Math., 98:185-191, 1991.

[11] J.-P. Roudneff and B. Sturmfels. Simplicials cells in arrangements and mutations in oriented matroids. Geom. Dedicata, 27:153-170, 1988.

[12] R.W. Shannon. Simplicial cells in arrangements of hyperplanes. Geom. Dedicata, 8:179-187, 1979. 